\title{
Pessoas com Diabetes Mellitus e a COVID-19
}

\author{
People with Diabetes Mellitus \\ and COVID-19
}

\section{Raimeyre Marques Torres ${ }^{1}$ (1) Marcela da Silva Souza ${ }^{2}$ (1)}

${ }^{1}$ Autora para correspondência. Universidade Federal da Bahia (Salvador), Bahia, Brasil. raimeyretorres@yahoo.com.br 2Universidade Federal da Bahia (Salvador). Bahia, Brasil. marcelas.s@msn.com

As doenças crônicas não-transmissíveis (DCNT) são as principais causas de morte e de incapacidade prematura no mundo sendo o diabetes mellitus (ou diabetes) a mais prevalente e responsável por $80 \%$ dessas mortes ${ }^{1}$. Mundialmente, mais de 425 milhões de pessoas tem diabetes, ou 8,8\% dos adultos de 20 a 79 anos, transformando-o num importante problema de saúde pública, devido aos custos associados ao tratamento, complicações microvasculares/macrovasculares e hospitalizações². No Brasil, de 2006 a 2019 a prevalência de diabetes cresceu de 5,5\% para $7,4 \%$, respectivamente 3 .

Em dezembro de 2019, uma série de casos de pneumonia de causa desconhecida surgiu em Wuhan, Hubei, China, com apresentações clínicas muito semelhantes à pneumonia viral. A análise profunda de sequenciamento de amostras do trato respiratório inferior indicou um novo coronavírus (CoV2), responsável pela síndrome respiratória aguda grave por coronavírus 2 (SARS-CoV-2; anteriormente conhecido como 2019-nCoV) e em fevereiro de 2020 foi designada doença de coronavírus 2019 (COVID-19)4.
O diabetes como uma comorbidade que complica e aumenta o número de óbitos nas doenças pulmonares ainda é pouco estudado. No entanto, o advento da pandemia da COVID-19 coloca-o como uma das principais comorbidades para o aumento no risco de complicações, hospitalizações e morte em pacientes contaminados pela COVID-1955. O estudo de Xiaobo Yang e colaboradores realizado na China ${ }^{6}$ reforça essa discussão, pois 7 (22\%) de um grupo de 32 pacientes internados em uma unidade de terapia intensiva com pneumonia por SARSCoV-2 que foram a óbito, tinham diabetes. Outros dois estudos com chineses ${ }^{7,8}$ também reconhecem o diabetes como uma comorbidade importante para piores resultados clínicos e desfechos fatais quando associado à COVID-19.

No Brasil não é diferente: dados do Ministério da Saúde demonstram que $70 \%$ dos óbitos por COVID-19 ocorreram em maiores de 60 anos e, $67 \%$ deles apresentavam pelo menos uma comorbidade, sendo o diabetes o segundo colocado com 1.123 óbitos . Na Bahia, segundo dados do boletim epidemiológico COVID-19, foram registrados 2.483 óbitos, sendo $6,74 \%$ deles em maiores de 70 anos, com uma taxa de comorbidades de 
$77,12 \%$ e o diabetes também ocupou o segundo lugar com $35,4 \%$ dos óbitos ${ }^{10}$.

Os mecanismos que tornam o diagnóstico de diabetes um risco para progressão e prognóstico dos indivíduos contaminados pela COVID-19 ainda não são totalmente explicados. Além do mais, considerandose a alta prevalência de doenças cardiovasculares $(D C V)$, obesidade e hipertensão em pacientes com diabetes, não se pode comprovar se essa doença contribui de forma independente para esse risco aumentado 5 . No entanto, estudo entre pacientes com COVID-19 e diabetes e sem outras comorbidades, revelou que eles são mais predispostos a uma tempestade inflamatória, levando a uma rápida deterioração do estado hemodinâmica geral em comparação com aqueles sem diabetes ${ }^{11}$.

Entretanto, além da predisposição, da síndrome da inflamação e da tempestade de citocinas, outros mecanismos potenciais podem aumentar a suscetibilidade à COVID-19 em pacientes com diabetes, tais como: 1) ligação celular de maior afinidade e entrada eficiente do vírus, 2) diminuição da depuração viral, 3) diminuição da função das células T e 5) presença de DCV 5 . O SARS-CoV2 se liga às células-alvo através da enzima convertora de angiotensina 2 (ECA2), usando-a como receptor de entrada e subregula frequentemente a sua expressão ${ }^{5}$. Dados sugerem que a expressão da ECA2 é aumentada no diabetes e o tratamento com inibidores da ECA aumenta a sua expressão, facilitando a infecção por COVID-1912. Os defeitos das funções das células $T$ e o excesso de produção de citocinas tipo 2 podem levar a uma deficiência no controle da replicação viral e a respostas pró-inflamatórias mais prolongadas, contribuindo para um prognóstico ruim em pacientes com COVID-19 ${ }^{13}$.

Sabendo-se que o curso clínico dos indivíduos com a COVID-19 e comorbidades, como o diabetes, tende a ser mais grave e complexo, podendo evoluir para o óbito, quais seriam essas complicações?

Estudo com 174 participantes com COVID-19, sendo 37 com diabetes e 137 sem diabetes demonstrou que as pessoas com diabetes apresentam níveis séricos de biomarcadores inflamatórios (interleucina-6, proteína $C$ reativa/PCR e ferritina sérica) e índice de coagulação (dímero $D$ ) mais elevados em comparação com aqueles $\operatorname{sem}^{11}$. Níveis significativamente mais alto de dímero $D$ e PCR também foram observados em outros estudos onde a presença de comorbidades como diabetes e hipertensão contribuíram para mortalidade elevada na COVID ${ }^{8,13}$.

Considerando-se a susceptibilidade e o maior risco de desfecho ruim para as pessoas com diabetes acometidas pelo SARS-CoV-25,11, medidas para proteger os indivíduos com essa comorbidade precisam ser implementadas, visando minimizar o impacto atual e futuro sobre esses indivíduos, o sistema de saúde e a economia ${ }^{14}$.

No entanto, estudo realizado na América do Sul e Central sobre o impacto da Covid-19 nas pessoas com diabetes identificou que mais de $68 \%$ dos entrevistados relataram escassez ou falta de medicamentos na eminência da pandemia e quase $58 \%$ referiram dificuldades no acesso aos serviços de saúde ${ }^{14}$.

Estudo realizado no Brasil revelou que a estratégia de distribuição de medicamentos e insumos médicos por 90 dias, a fim de evitar viagens mensais das pessoas com diabetes aos serviços de saúde, foi eficaz para apenas $21 \%$ dos $64,5 \%$ que receberam medicamentos e insumos do SUS ${ }^{15}$. Por isso, é importante investir em recursos humanos e materiais para a saúde, visando reverter essa situação, implementando medidas para garantir o acesso a medicamentos e serviços de saúde, com o objetivo de prevenir o colapso do sistema de saúde causado pela infecção pelo SARS-CoV-2 em pessoas de maior risco ${ }^{14}$.

No que se referem aos cuidados em âmbito hospitalar, o diagnóstico e controle do diabetes devem ser investigados nos pacientes admitidos para tratamento da COVID-19 e devem orientar a tomada de decisão sobre hospitalização, suporte respiratório precoce e terapia adequada para melhorar a mortalidade.

Sabendo de seu maior risco, especialmente quando os níveis glicêmicos se apresentam fora do alvo, medidas de proteção e estratégias para facilitar o tratamento ideal devem ser adotados ${ }^{14}$. Assim, medidas prioritárias para garantir o alvo glicêmico, o manejo interdisciplinar de casos graves e o acompanhamento clínico pós-alta, podem contribuir para a saúde geral dos indivíduos com diabetes durante e após a infecção por SARS-CoV-2. 


\section{Referências}

1. Sociedade Brasileira de Diabetes. Diretrizes da sociedade brasileira de diabetes 2019-2020/SBD. São Paulo; Clannad; 2020.

2. International Diabetes Federation/IDF. Clinical Guidelines Task Force. Global guideline for Type 2 diabetes. Brussels: International Diabetes Federation. [Internet]. 2020. Disponível em: https:// www.iapb.org/wp-content/uploads/Global-Guideline-for-Type-2Diabetes-IDF-2012.pdf

3. Ministério da Saúde. Secretaria de Vigilância em Saúde. Departamento de Vigilância de doenças e agravos não transmissíveis e Promoção da Saúde. Vigilância de fatores de risco e proteção para doenças crônicas por inquérito telefônico [Internet]. 2019. Disponível em: https://www.saude.gov.br/ images/pdf/2020/April/27/vigitel-brasil-2019-vigilancia-fatoresrisco.pdf

4. Chen Y, Liu Q, Guo D. Emerging coronaviruses: Genome structure, replication, and pathogenesis. J Med Virol. 2020;92(4):418-423. doi: 10.1002/jmv.25681

5. Muniyappa R, Gubbi S. COVID-19 pandemic, coronaviruses, and diabetes mellitus. Am J Physiol Endocrinol Metab. 2020;318(5):736741. doi: 10.1152 /ajpendo.00124.2020

6. Yang X, Yu Y, Xu J, Shu H, Xia J, Liu H et al. Clinical course and outcomes of critically ill patients with SARS-CoV-2 pneumonia in Wuhan, China: a single-centered, retrospective, observational study. Lancet Respir Med. 2020;8(20):475-81. doi: 10.1016/S22132600(20)30079-5

7. Guan WJ, Liang WH, Zhao Y, Liang HR, Chen ZS, Li YM et al. Comorbidity and its impact on 1590 patients with COVID-19 in China: a nationwide analysis. Eur Respir J. 2020;55(5):2000547. doi: $10.1183 / 13993003.00547-2020$
8. Zhang J, Dong X, Cao YY, Yuan YD, Yang YB, Yan YQ et al. Clinical characteristics of 140 patients infected by SARS-CoV-2 in Wuhan, China. Allergy. 2020;75(7):1730-1741. doi: $10.1111 /$ all.14238

9. Ministério da Saúde. Secretaria de Vigilância em Saúde. Dados epidemiológicos do dia 17/05 [Internet]. 2020. Disponível em: http://saude.gov.br/images/pdf/2020/June/25/Boletimepidemiologico-COVID-19-2.pdf

10. Secretaria de Saúde do Estado da Bahia - SESAB. Boletim Epidemiológico Covid-19. Secretaria de Saúde da Bahia (Sesab) n 110 - julho de 2020 [Internet]. 2020. Disponível em: http:// www.saude.ba.gov.br/wp-content/uploads/2020/07/BOLETIM_ ELETRONICO_BAHIAN_108_10072020-1-2.pdf

11. Guo W, Li M, Dong Y, Zhou H, Zhang Z, Tian C et al. Diabetes is a risk factor for the progression and prognosis of COVID-19. Diabetes Metab Res Rev. 2020:e3319. doi: 10.1002/dmrr.3319

12. Fang L, Karakiulakis G, Roth M. Are patients with hypertension and diabetes mellitus at increased risk for COVID-19 infection? Lancet Respir Med. 2020;8(4):e21. doi: 10.1016/S22132600(20)30116-8

13. Zhou F, Yu T, Du R, Fan G, Liu Y, Liu Z et al. Clinical course and risk factors for mortality of adult inpatients with COVID-19 in Wuhan, China: a retrospective cohort study. Lancet 2020;395:1054-62. doi: 10.1016/S0140-6736(20)30566-3

14. Barone MTU, Villarroel D, Luca PV, Harnik SB, Lima BLS, Pineda-Wieselberg RJ et al. COVID-19 Impact on People with Diabetes in the South and Central America. Diabetes Res Clin Pract. 2020;166:108301. doi: 10.1016/j.diabres.2020.108301

15. Barone MTU, Harnik SB, Luca PV, Lima BLS, Pineda-Wieselberg RJ, Ngongo B et al. The impact of COVID-19 on people with diabetes in Brazil. Diabetes Res Clin Pract 2020;166:108304. doi: 10.1016/j.diabres.2020.108304 